\title{
Use of Crystallography and Molecular Modeling for the Inhibition of the Botulinum Neurotoxin A Protease
}

Lewis D. Turner, Alexander L. Nielsen, Lucy Lin, Antonio J. Campedelli, Nicholas R. Silvaggi, Jason S. Chen, Amanda E. Wakefield, Karen N. Allen, and Kim D. Janda*

\begin{abstract}
Botulinum neurotoxins (BoNTs) are extremely toxic and have been deemed a Tier 1 potential bioterrorism agent. The most potent and persistent of the BoNTs is the "A" serotype, with strategies to counter its etiology focused on designing small molecule inhibitors of its light chain (LC), a zincdependent metalloprotease. However, the successful structure-based drug design (SBDD) of inhibitors has been confounded as the LC is highly flexible with significant morphological changes occurring upon inhibitor binding. To achieve greater success, previous and new co-crystal structures were evaluated from the standpoint of inhibitor enantioselectivity and their effect on active-site morphology. Based upon these structural insights, a previously unknown hydrophobic sub-pocket adjacent to the S1' site was discovered and used to design inhibitors that take advantage of $\pi-\pi$ stacking interactions. Structure-activity relationships (SARs) were defined and X-ray crystal structures and docking models were examined to rationalize the observed potency differences between inhibitors. The structure-guided design of inhibitors that utilize both the cryptic hydrophobic sub-pocket and the essential aspects to enantiomer selectivity provide a new avenue for ablating BoNT/A protease activity.
\end{abstract}

KEYWORDS: BoNT/A, SBDD, enantioselectivity, hydroxamate, SARs, pi-pi stacking

BoNTs possess incredible potency and are among the most toxic agents known to humankind. Seven serotypes $(A-G)$ have been identified ${ }^{1}$ with serotype A exhibiting the highest incidence rate of botulism within the United States. ${ }^{2}$ This is attributed to its remarkable toxicity (intravenous $\mathrm{LD}_{50}=1-$ $2 \mathrm{ng} / \mathrm{kg})^{3}$ and persistence within human tissue $\left(t_{1 / 2}=\right.$ months to years $) .{ }^{4}$ These properties, coupled with its ease of production and dissemination, has led to categorization by the Centers for Disease Control and Prevention (CDC) as a Tier 1 potential bioterrorism agent. ${ }^{5}$ BoNTs are produced by a species of diverse anaerobic bacteria known as Clostridium botulinum, and also pose a risk of foodborne botulism from incorrect handling of food products, particularly canned products. ${ }^{6,7}$

The remarkable potency of BoNT/A has been utilized in a multitude of therapeutic and cosmetic applications in which aberrant muscular spasticity is implicated, with several Food and Drug Administration (FDA)-approved formulations available for various and limited indications. ${ }^{8}$ Debilitating disorders such as cerebral palsy, ${ }^{9}$ chronic migraines, ${ }^{10}$ and sialorrhea, ${ }^{11}$ and cosmetic imperfections such as facial wrinkles, ${ }^{12}$ have been successfully treated using BoNT/A formulations. The prolific use of BoNT/A for these therapeutic applications comes with associated risks; misuse, overdose or poor injection technique of BoNT/A has established side-effects such as spread from the injection site, iatrogenic botulism, and respiratory compromise. ${ }^{13}$ 
The effects associated with BoNT/A intoxication arise from its ability to prevent exocytotic vesicle fusion at neuronal termini, thus preventing neurotransmitter release and disrupting normal muscular function which results in the flaccid paralysis characteristic of botulism. ${ }^{14}$ The $150 \mathrm{kDa}$ BoNT/A holotoxin consists of a heavy chain (HC, $100 \mathrm{kDa})$ and a light chain (LC, $50 \mathrm{kDa}$ ) that are connected by a single disulfide bond. With facilitation from the $\mathrm{HC}$ binding to receptor surface sites, the LC, a zinc-dependent metalloprotease, is translocated to the cytosol. ${ }^{15}$ Once internalized, the LC selectively cleaves synaptosomal-associated protein 25 (SNAP-25), a crucial component of the soluble N-ethylmaleimide sensitive factor (NSF) attachment protein receptor (SNARE) complex that is responsible for vesicular fusion to the synaptosomal membrane. ${ }^{16}$ Current post-intoxication treatments for BoNT/A are limited to antibody-based antitoxins which have a short therapeutic window (12-24 hr post-exposure) owing to their inability to enter the neuronal compartment. ${ }^{17}$ This highlights an unmet need for new post-intoxication treatments for BoNT/A that can intercept internalized LC activity and match toxin persistence; one such strategy is the development of small-molecule inhibitors.

Currently, several examples of small-molecule inhibitors for the BoNT/A LC have been identified and primarily function through active-site zinc ion chelation. ${ }^{18,}{ }^{19}$ Some of these inhibitors (Figure 1A) have been co-crystallized within the LC and distinct morphological changes in the S1' subsite have been observed (Figure 1B/C). Lipophilic inhibitors 2,4-dichlorocinnamic hydroxamic acid (DCHA) and adamantane-based inhibitor 1 possess almost identical binding poses and occupy the S1' subsite, a small hydrophobic cleft comprised of residues Phe194, Ile161, Asp370 and Phe369. The phenyl ring of DCHA forms an offset $\pi-\pi$ stack with Phe194 with the 370 loop adopting a Phe369in-Asp370-out conformation with Phe369 forming a partial lid above the inhibitors. ${ }^{20,21}$ Conversely, hydrophilic inhibitor L-arginine-based hydroxamate 2 causes a conformational inward flip of Asp370, forming H-bond interactions with the guanidinium moiety of 2.22, ${ }^{23}$ Moreover, Phe194 has also rotated to form a cation-pi interaction with $\mathbf{2}$. Additional examples of small-molecule hydroxamate inhibitors have also outlined a high degree of flexibility within the S1' region of the BoNT/A LC. ${ }^{24}$

The advancement of structural information for the BoNT/A LC has enabled structure-guided design of enantioselective chiral inhibitors as outlined by Stowe et al. ${ }^{25}$ Analysis of the active-site binding pose of DCHA outlined two proximal water molecules involved in an H-bonding network with amino acid residues His223, Glu351, and Arg363 (Figure 1D). With the aid of molecular modeling, compounds 3 and $\mathbf{4}$ were designed with two purposes in mind: 1) extend additional chemical composition into the polar space of the active site and 2) investigate the effect of chiral inhibitors upon BoNT/A LC inhibition. ${ }^{25}$ Specifically, the hydroxyethyl appendage was hypothesized to displace one of the ordered water molecules within the enzyme active site. Moreover, previous BoNT/A LC inhibition data revealed a $\sim 4$ and 21-fold preference for the $R$ enantiomer over the $S$ enantiomer of 3 
and 4, respectively. ${ }^{25}$ To investigate this selectivity, we have determined the co-crystal structure of racemic 3 bound within the BoNT/A LC (Figure 1E).

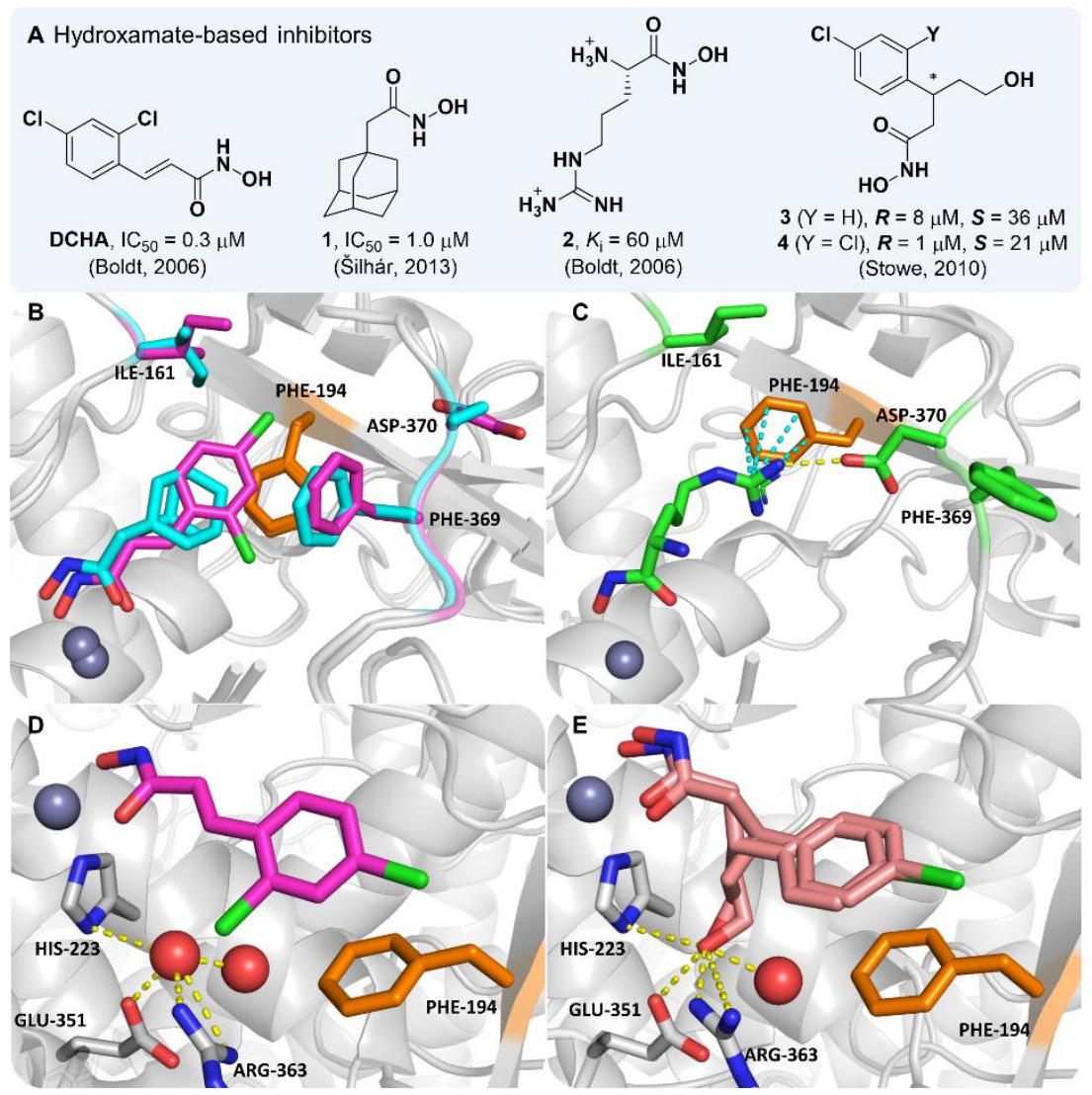

Figure 1: (A) Hydroxamate-based inhibitors of the BoNT/A LC. Values for 3 and 4 are $\mathrm{IC}_{50}$. (B) Overlay of Xray crystal structures of DCHA (purple, PDB 2IMA) and $\mathbf{1}$ (cyan, PDB 4HEV) bound within the active site of the LC. The 370 loop adopts an Phe369-in-Asp370-out conformation. (C) X-ray crystal structure of 2 (green, PDB 2IMB) bound within the active site of the LC. The 370 loop adopts an Phe369-out-Asp370-in conformation. Hydrogen bonds and cation- $\pi$ interactions are outlined in yellow and cyan dashed lines, respectively. Alternative view of DCHA bound within the active site of the LC. Structural water molecules are outlined in red spheres with nearby polar contacts indicated by yellow dashed lines. (E) Co-crystal structure of $\mathbf{3}$ bound within the active site of the LC (PDB 7N18) determined at a resolution of $2.1 \AA$. Both $R$ and $S$ enantiomers are shown. The hydroxyethyl group has displaced one of the water molecules observed in the structure of the complex with DCHA. Nearby polar contacts are indicated by yellow dashed lines.

Although the inhibitor could be unambiguously placed within the active-site, electron density at the sterogenic center of $\mathbf{3}$ was poorly defined (Figure S1). As we crystallized with the racemic mixture, and the enantiomers had comparable potencies, we concluded that each enantiomer had similiar occupancies within the LC crystal lattice and an average of the two structures was observed. Despite this, electron density for the phenyl ring, the hydroxamate, and the hydroxyethyl appendage was well defined, with the hydroexethyl $\mathrm{OH}$ group displacing one water molecule (Figure 1D) to form an H-bond network with residues His223, Glu351, and Arg363. Notably, the inclusion of the 2-chloro substituent in 4 greatly improved enantiomer selectivity; ${ }^{25}$ evaluation of the binding poses of both 
DCHA and 3 show the phenyl ring making a $\pi-\pi$ stacking interaction with Phe194. Based on these insights, we posit that the 2-chloro substituent of $\mathbf{4}$ acts as a "molecular brake" and reduces the free rotation of the phenyl ring, causing a greater chiral effect at the stereocenter.

Recently, a central focus of the development of BoNT/A LC inhibitors has been upon the irreverible inhibition of the enzyme. Success in this area has been found through a bifunctional strategy, wherein coordination to the active-site zinc ion allows proximal positioning of a pendent electrophilic warhead for the simultaneous irreversible modification of a non-catalytic cysteine. ${ }^{26,27}$ Compound $\mathbf{5}$, possessing an electrophilic methanethiosulfonate (MTS) warhead and the same branched pharmacophore as $\mathbf{3 / 4}$, exhibited modest irreversible inhibition against the BoNT/A LC with a $k_{\text {inact }} / K_{\mathrm{i}}$ value of $19.4 \mathrm{M}^{-1} \cdot \mathrm{s}^{-1}$ (Figure 2A). ${ }^{26}$ As proof-of-concept for this covalent approach, compound $\mathbf{6}$ was synthesized as a non-covalent control and was crystallized in complex with the BoNT/A LC (Figure 2C) ${ }^{26}$ This structure revealed a new cryptic hydrophobic sub-pocket adjacent to the main S1' binding pocket that was absent in the crystal structure of DCHA bound to the BoNT/A LC (Figure 2B). The $\pi$ $\pi$ stacking interaction observed between the 2,4-dichlorophenyl ring in DCHA and the side chain of Phe194 is not present upon binding of $\mathbf{6}$. The induced fit caused by the alkyl moiety results in a large 
shift and rotation of the side chain of Phe194, and together with Phe163, both form the edges of the new hydrophobic sub-pocket effectively 'sandwiching' the alkyl chain.

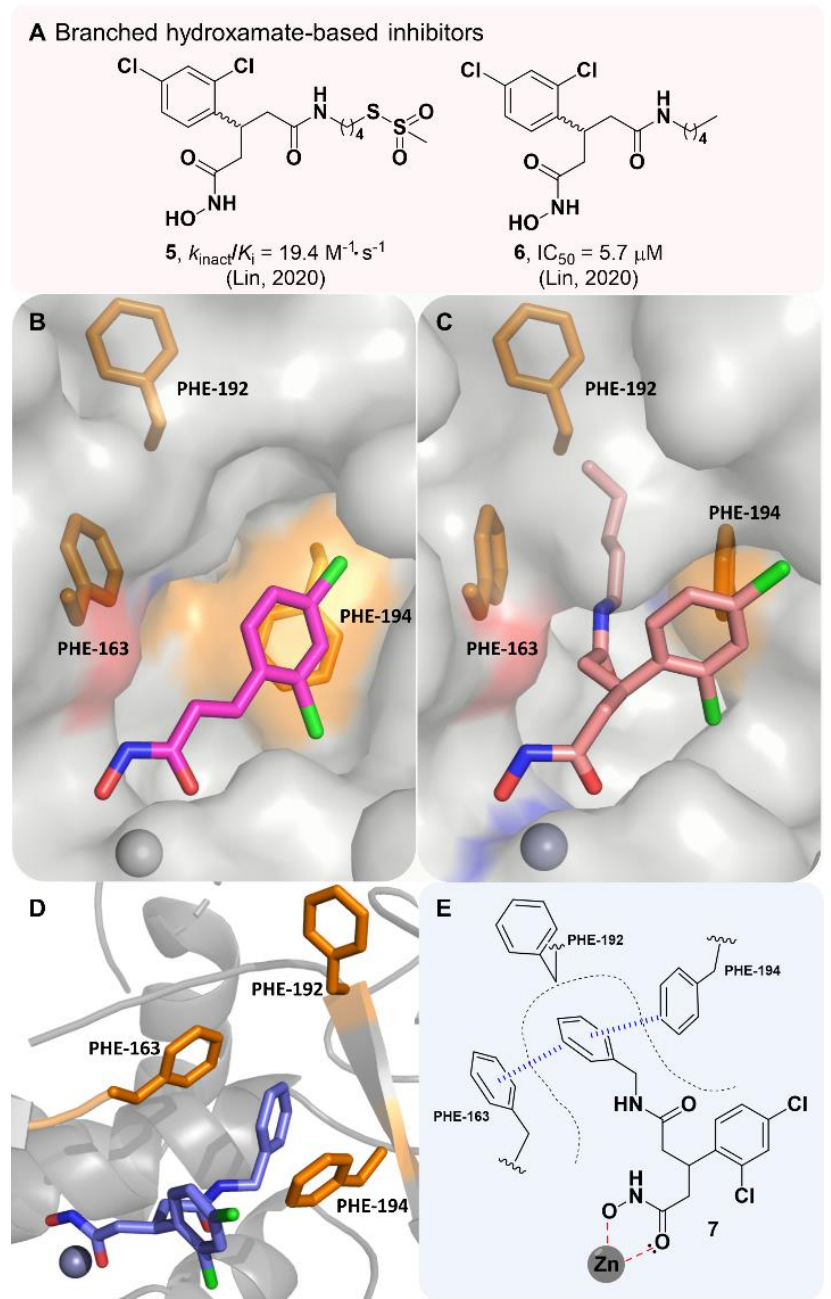

Figure 2: (A) Branched hydroxamate-based inhibitors of the BoNT/A LC. (B) Alternative view of DCHA (purple) bound with the LC. The 2,4-dichlorocinnamic phenyl ring and Phe194 form an offset face-to-face $\pi-\pi$ stack. (C) X-ray crystal structure of $\mathbf{6}$ bound in the active site of the LC (PDB 6XCF). Accommodation of the alkyl moiety in the hydrophobic sub-pocket results in disruption of the face-to-face $\pi$ - $\pi$ stack between the 2,4-dichlorocinnamic phenyl ring and Phe194. (D) Docking pose of 7 bound to the BoNT/A LC. (E) 2D representation of the predicted binding mode of $\mathbf{7}$ in the BoNT/A LC active-site. Coordination of the hydroxamate and $\pi-\pi$ stacking interactions are outlined in red and blue, respectively.

Formation of this cryptic hydrophobic sub-pocket has not previously been observed; to quantify the size of this additional binding pocket and the main S1' pocket, we used Dpocket, a module within the Fpocket suite, to calculate pocket volumes (Table S1) for DCHA, 1-3 and 6. ${ }^{28,}{ }^{29}$ Dpocket defines the pocket using atoms within $4 \AA$ of the ligand, thus it is expected that larger ligands give rise to larger binding pockets. With the exception of $\mathbf{2}$, the results clearly reflected this expectation, with $\mathbf{6}$ filling additional sub-pockets within the active site compared to other ligands that only occupied the S1' pocket. Compound $\mathbf{2}$ is the smallest ligand but had a comparable pocket size to that of $\mathbf{6}$. Upon visual 
inspection of the binding poses of DCHA and 2, Pro69 exhibited a large shift toward solvent, resulting in a larger binding pocket for $\mathbf{2}$ (Figure $\mathbf{S 2}$ ).

We envisioned that we could design inhibitors based upon the branched pharmacophore of $\mathbf{6}$ to take advantage of this unexplored, cryptic, hydrophobic sub-pocket. Specifically, we postulated that we could create a tripartite $\pi$ - $\pi$ stacking interaction with Phe163/194 and a phenyl ring by replacement of the alkyl chain in $\mathbf{6}$. To bolster this hypothesis, we conducted docking studies of the benzylamine-based derivative 7 (Figure 2D). As posited, the benzylamine ring adopted a pose which occupied the hydrophobic sub-pocket with both Phe163 and 194 forming offset $\pi-\pi$ stacking interactions. Using this enabled logic, we designed a series of aniline- and benzylamine-based derivatives (Figure 3). The design included potential hydrophobic/hydrogen bonding contacts derived through either methyl/ethyl groups as well as $\mathrm{OH}$ and $\mathrm{NH}_{2}$ moieties that were strategically placed on the aromatic ring.

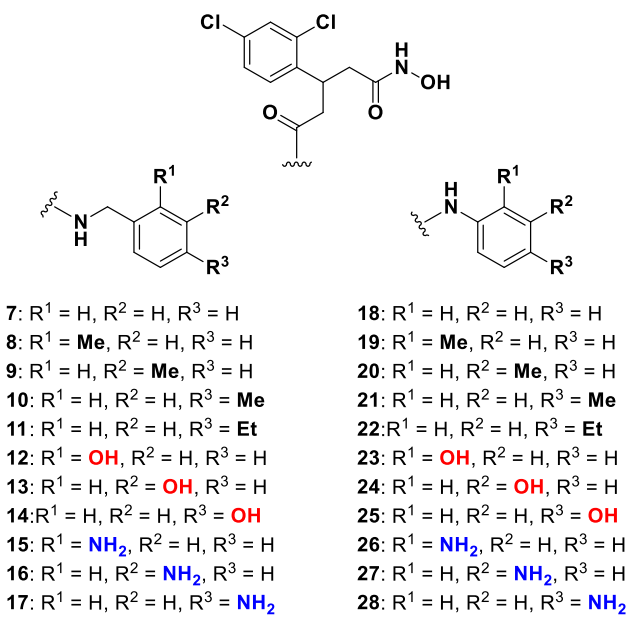

Figure 3: Aniline- and benzylamine-based target library.

Synthesis of these target compounds 7-28 (Scheme 1) began with ring opening of previously synthesized cyclic anhydride $2 \mathbf{2 9}^{26}$ with $O$-tetrahydropyran (THP)-protected hydroxylamine to afford the carboxylic acid intermediate $\mathbf{3 0}$ in a moderate yield. Acid $\mathbf{3 0}$ was subjected to amide coupling conditions with various benzylamines to afford protected hydroxamates $(\mathbf{3 1})$ that were then deprotected using pyridinium $p$-toluenesulfonate (PPTS) to yield the final target compounds. Notably, amide coupling conditions for $\mathbf{3 0}$ using less-reactive/ortho-substituted aniline reagents resulted in no formation of the desired product. Fortunately, a change in order of steps (Route 2) reacting aniline reagents with 29 directly to give carboxylic acids (32) was successful. Furthermore, optimization of work-up procedures allowed both the amide coupling and deprotection step to be combined allowing rapid access to the final compounds $\mathbf{7 - 2 8}$. Further details on the synthetic routes employed can be found in SI-4.0. 


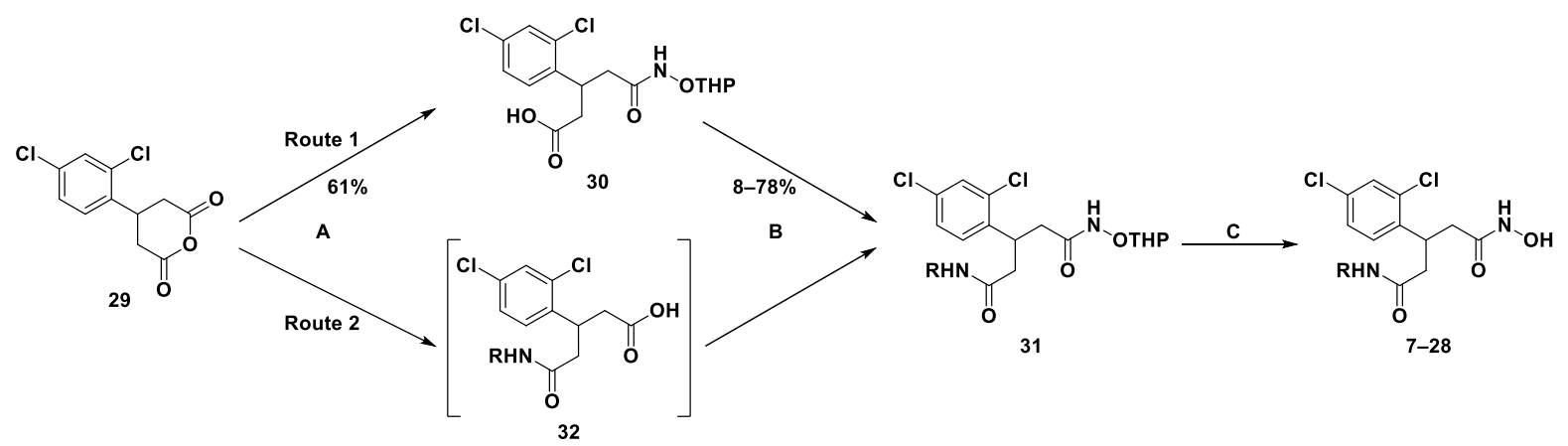

Scheme 1: General route to benzylamine- and aniline-based final compounds. 31 and 32 represent multiple intermediates. Reagents and conditions: (A) $\mathrm{RNH}_{2}, \mathrm{CHCl}_{3}$ or $\mathrm{CH}_{2} \mathrm{Cl}_{2}$, r.t; (B) $\mathrm{RNH}_{2}$, HATU or EDC, DIPEA, DMF, r.t; (C) PPTS, EtOH, $65{ }^{\circ} \mathrm{C}$ or $10 \%$ TFA- $\mathrm{CH}_{2} \mathrm{Cl}$, r.t.

Having established solid synthetic routes, hydroxamates 7-28 were evaluated for BoNT/A LC inhibition (Table 1) using a previously described ${ }^{26}$ fluorescence resonance energy transfer (FRET)based assay that uses a truncated form of the BoNT/A LC (amino acids 1-425) and a SNAPtide substrate. ${ }^{30,31}$ Compounds were pre-incubated for 30 minutes at various concentrations with $10 \mathrm{nM}$ BoNT/A LC followed by addition of $4 \mu \mathrm{M}$ substrate with reaction progress fluorescently monitored at ex/em 490/523 nm.

Table 1: $\mathrm{IC}_{50}$ values for 7-28 when evaluated against the FRET-based SNAPtide assay. See SI-6.1 for dose response curves.

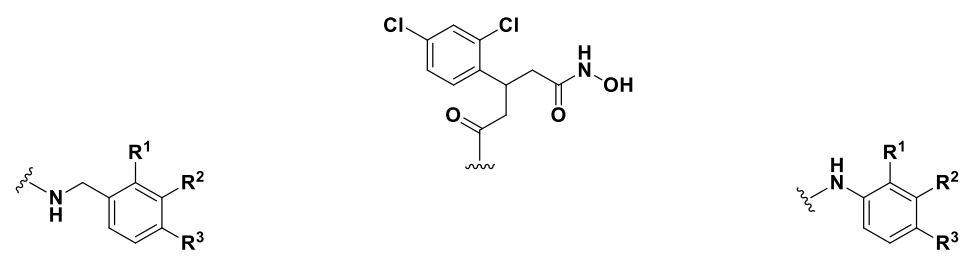

\begin{tabular}{ccccc|ccccc}
\hline Compound & $\mathbf{R}_{\mathbf{1}}$ & $\mathbf{R}_{\mathbf{2}}$ & $\mathbf{R}_{\mathbf{3}}$ & $\mathbf{I C}_{\mathbf{5 0}} \mathbf{a}^{\mathbf{2}} \mathbf{\text { SD }}(\boldsymbol{\mu} \mathbf{M})$ & Compound & $\mathbf{R}_{\mathbf{1}}$ & $\mathbf{R}_{\mathbf{2}}$ & $\mathbf{R}_{\mathbf{3}}$ & $\mathbf{I C}_{\mathbf{5 0}}^{\mathbf{a}} \pm \mathbf{S D}(\boldsymbol{\mu M})$ \\
\hline $\mathbf{7}$ & $-\mathrm{H}$ & $-\mathrm{H}$ & $-\mathrm{H}$ & $4.0 \pm 0.2$ & $\mathbf{1 8}$ & $-\mathrm{H}$ & $-\mathrm{H}$ & $-\mathrm{H}$ & $0.8 \pm 0.2$ \\
$\mathbf{8}$ & $-\mathrm{Me}$ & $-\mathrm{H}$ & $-\mathrm{H}$ & $1.9 \pm 0.3$ & $\mathbf{1 9}$ & $-\mathrm{Me}$ & $-\mathrm{H}$ & $-\mathrm{H}$ & $1.6 \pm 0.2$ \\
$\mathbf{9}$ & $-\mathrm{H}$ & $-\mathrm{Me}$ & $-\mathrm{H}$ & $2.0 \pm 0.1$ & $\mathbf{2 0}$ & $-\mathrm{H}$ & $-\mathrm{Me}$ & $-\mathrm{H}$ & $3.2 \pm 0.2$ \\
$\mathbf{1 0}$ & $-\mathrm{H}$ & $-\mathrm{H}$ & $-\mathrm{Me}$ & $3.4 \pm 0.3$ & $\mathbf{2 1}$ & $-\mathrm{H}$ & $-\mathrm{H}$ & $-\mathrm{Me}$ & $2.7 \pm 0.1$ \\
$\mathbf{1 1}$ & $-\mathrm{H}$ & $-\mathrm{H}$ & $-\mathrm{Et}$ & $9 \pm 1$ & $\mathbf{2 2}$ & $-\mathrm{H}$ & $-\mathrm{H}$ & $-\mathrm{Et}$ & $3.8 \pm 0.1$ \\
$\mathbf{1 2}$ & $-\mathrm{OH}$ & $-\mathrm{H}$ & $-\mathrm{H}$ & $11 \pm 2$ & $\mathbf{2 3}$ & $-\mathrm{OH}$ & $-\mathrm{H}$ & $-\mathrm{H}$ & $1.0 \pm 0.1$ \\
$\mathbf{1 3}$ & $-\mathrm{H}$ & $-\mathrm{OH}$ & $-\mathrm{H}$ & $12.1 \pm 0.8$ & $\mathbf{2 4}$ & $-\mathrm{H}$ & $-\mathrm{OH}$ & $-\mathrm{H}$ & $3.3 \pm 0.7$ \\
$\mathbf{1 4}$ & $-\mathrm{H}$ & $-\mathrm{H}$ & $-\mathrm{OH}$ & $21 \pm 4$ & $\mathbf{2 5}$ & $-\mathrm{H}$ & $-\mathrm{H}$ & $-\mathrm{OH}$ & $3.9 \pm 0.5$ \\
$\mathbf{1 5}$ & $-\mathrm{NH} 2$ & $-\mathrm{H}$ & $-\mathrm{H}$ & $36 \pm 9$ & $\mathbf{2 6}$ & $-\mathrm{NH} 2$ & $-\mathrm{H}$ & $-\mathrm{H}$ & $4.6 \pm 0.2$ \\
$\mathbf{1 6}$ & $-\mathrm{H}$ & $-\mathrm{NH} 2$ & $-\mathrm{H}$ & $23 \pm 1$ & $\mathbf{2 7}$ & $-\mathrm{H}$ & $-\mathrm{NH} 2$ & $-\mathrm{H}$ & $8.9 \pm 0.4$ \\
$\mathbf{1 7}$ & $-\mathrm{H}$ & $-\mathrm{H}$ & $-\mathrm{NH}_{2}$ & $26 \pm 5$ & $\mathbf{2 8}$ & $-\mathrm{H}$ & $-\mathrm{H}$ & $-\mathrm{NH}_{2}$ & $14 \pm 2$
\end{tabular}

${ }^{\text {a }}$ Average of at least three independent experiments.

The initial target hydroxamate 7 had an $\mathrm{IC}_{50}$ value of $4.0 \mu \mathrm{M}$, thus showing no real improvement when compared to the alkyl-containing counterpart $6\left(\mathrm{IC}_{50}=5.7 \mu \mathrm{M}\right)$. This suggested that the hypothesized $\pi-\pi$ stacking interaction with residues Phe163 and 194 did not provide significant 
binding stabilization or was unlikely to be occurring. Addition of a methyl group in the ortho (8) and meta (9) position produced a $\sim 2$-fold increase in potency whereas the para substituted compound $\mathbf{1 0}$ exhibited minimal effect on the potency. Of note, addition of an ethyl group (11) in the para position resulted in a $\sim 2$-fold loss in potency when compared to unsubstituted benzylamine 7 . With the docked structure of $\mathbf{7}$ (Figure 2D) in mind, this suggested that $\mathbf{1 1}$ may be too large, potentially clashing with Phe192 deep within the hydrophobic sub-pocket.

Hydroxamates 12-17 were designed to explore hydrogen bonding potential within the hydrophobic sub-pocket. Substitution at either the ortho, meta, or para position with polar $\mathrm{OH}$ and $\mathrm{NH}_{2}$ groups for benzylamine-based inhibitors resulted in a large decrease in potency. This was readily interpreted as repulsive interactions between the hydrophobic residues that line the sub-pocket and the appended polar groups, and that H-bonding contacts may be untenable. Unsubstituted aniline $\mathbf{1 8}$ exhibited a sub-micromolar $\mathrm{IC}_{50}$ value of $0.8 \mu \mathrm{M}$, a $\sim$ - and 5-fold increase over 6 and 7, respectively. This indicated optimum positioning and alignment of the aniline ring for the predicted $\pi$ - $\pi$ stacking interaction with Phe163/194. To further explore these differences, we conducted docking of $\mathbf{1 8}$ and compared the docked pose to that of $\mathbf{7}$ (Figure 4).

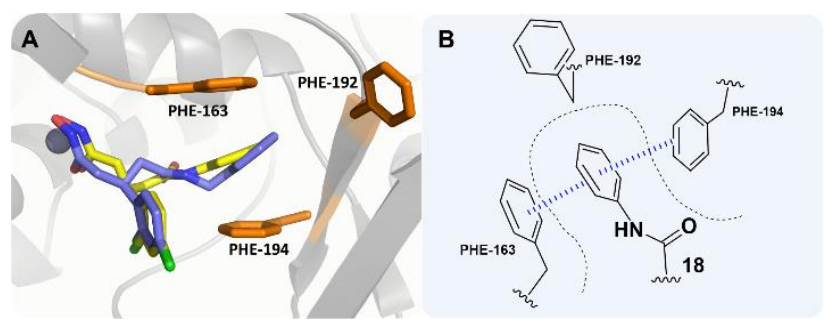

Figure 4: (A) Docking poses of 7 (blue) and 18 (yellow) bound within the BoNT/A LC. (B) 2D representation of the predicted $\pi$-sandwich interaction between Phe163 and 194 and the aniline ring of 18. $\pi$ - $\pi$ stacking interactions are outlined in blue.

The aniline phenyl ring in $\mathbf{1 8}$ was predicted to form a double offset $\pi-\pi$ interaction with Phe163 and 194 (Figure S3) and exhibited a more planar orientation with respect to the phenylalanine ring systems than what was observed for $\mathbf{7}$. The additional methylene group present in $\mathbf{7}$ was foreseen to disrupt the planarity of the $\pi-\pi$ stacking interactions and could account for the lower potency observed for the benzylamine-based compounds. Contrary to the benzylamine series, addition of a methyl group in the ortho (19), meta (20) and para (21) positions resulted in a loss of potency compared to unsubstituted aniline 18. Ethyl-containing aniline 22 also exhibited a loss in potency but was still superior to its benzylamine counterpart 11. The reduced extendibility of the aniline compounds may allow for larger groups in the para position to be better accommodated deep within the hydrophobic sub-pocket. Interestingly, compounds 23-28 exhibited improved potency when compared to their benzylamine counterparts 12-17. This inferred that there was less repulsion between hydrophobic residues and the polar groups present on the aniline phenyl ring. As might be posited from the docking models (Figure 4), 7 was expected to extend further into the hydrophobic sub-pocket than 18; hence, 
the polar groups would be better tolerated when placed towards the entrance of the hydrophobic subpocket. This premise was bolstered by the superior potency of ortho-substituted compounds 23 and 26 when compared to their meta (24 and 27) and para ( 25 and 28) substituted analogues. However, the loss in potency for phenol/dianiline derivatives (23-28) when compared to $\mathbf{1 8}$ meant that it was unlikely any H-bonding contacts formed between the inhibitor and enzyme. In summary, aniline-based molecules 18-28 exhibited superior inhibitory potency when compared to benzylamine-based structures $\mathbf{7 - 1 7}$, which we propose is facilitated through $\pi$ - $\pi$ stacking interactions with the aniline phenyl ring and Phe163/194.

To further investigate the effect of chirality upon inhibitor binding, we employed chiral supercritical fluid chromatography (SFC) to separate enantiomers of the three best inhibitors 18, 19 and 23. This approach allowed us rapid access to enantiopure material without undertaking the cumbersome asymmetric synthetic approach outlined previously, ${ }^{25}$ details pertaining to SFC are outlined in SI-4.4. Relative stereochemistry of enantiomers was assigned through their specific rotation of plane-polarized light and their BoNT/A LC inhibition assessed using the FRET-based assay (Table 2).

Table 2: $\mathrm{IC}_{50}$ values for enantiopure compounds $\mathbf{1 8}, \mathbf{1 9}$ and 23.
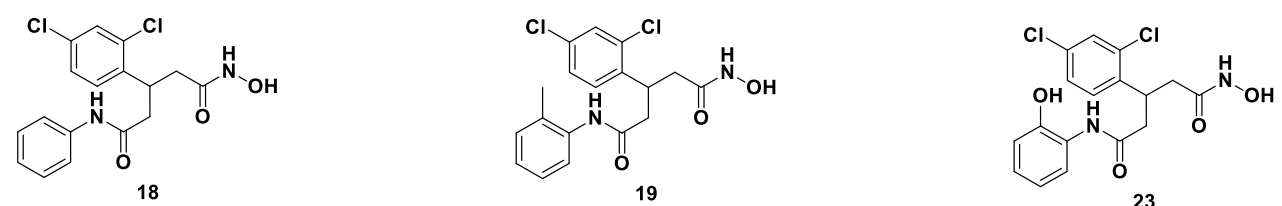

\begin{tabular}{|c|c|c|c|c|c|}
\hline Compound & $\mathrm{IC}_{50}{ }^{\mathrm{a}} \pm \mathrm{SD}(\mu \mathrm{M})$ & Compound & $\mathrm{IC}_{50}{ }^{\mathrm{a}} \pm \mathrm{SD}(\boldsymbol{\mu M})$ & Compound & $\mathrm{IC}_{50}{ }^{\mathrm{a}} \pm \mathrm{SD}(\mu \mathrm{M})$ \\
\hline$(-) 18$ & $1.9 \pm 0.4$ & $(-) 19$ & $1.6 \pm 0.2$ & $(-) 23$ & $1.5 \pm 0.1$ \\
\hline$(+) 18$ & $0.8 \pm 0.2$ & $(+) 19$ & $1.2 \pm 0.2$ & $(+) 23$ & $0.6 \pm 0.1$ \\
\hline
\end{tabular}

${ }^{a}$ Average of three independent experiments.

Differences in inhibitory potency were observed, albeit minimal, with the $(+)$ configuration being the preferred enantiomer with hydroxamates $\mathbf{1 8}$ and $\mathbf{2 3}$ exhibiting sub micromolar potency. In contrast these hydroxamates did not exhibit the same magnitude of enantiomer selectivity that was observed for 3 and $4 .^{25}$ The lack of the hydroxyethyl moiety, and subsequent loss of the H-bond network with amino acids His223, Glu351, and Arg363 (Figure 1E), for inhibitors 18, 19 and 23 suggests that this interaction is crucial for the development of exquisite enantioselective inhibitors of BoNT/A LC using this pharmacophore. The fluid morphology of the BoNT/A LC active site, coupled with the fact that both groups flanking the stereocenter are aromatic, suggests that both ring systems may have the capacity to occupy both the main S1' pocket and the hydrophobic sub-pocket.

In summary, we have designed, synthesized and biologically evaluated a comprehensive series of chiral benzylamine- and aniline-based inhibitors of the BoNT/A LC. With the aid of co-crystal structures and docking, molecules were designed to take advantage of a $\pi-\pi$ stacking interaction to improve inhibitor potency. Aniline-based hydroxamates possessed superior potency compared to the 
benzylamine-based hydroxamates, which was attributed to a more planar orientation of the $\pi-\pi$ stacking interaction, as predicted by docking. Moreover, the co-crystal structure of $\mathbf{3}$ has revealed that displacement of an active-site water molecule by the hydroxyethyl moiety is crucial to enantiomer selectivity. We envision that both occupation of the hydrophobic sub-pocket by aniline functionalities and the crucial H-bond network interaction obtained from the hydroxyethyl moiety can be combined into one entity that will not only possess improved potency but also greater enantioselectivity; work on such compounds is currently being conducted.

\section{Associated Content}

The Supporting Information is available free of charge at:

Supplementary figures, supplementary tables, biological materials and methods, chemistry experimental, compound dose response curves, compound HPLC chromatograms and NMR spectra.

\section{Author Information}

\section{Corresponding Author}

Kim D. Janda - Departments of Chemistry and Immunology, The Skaggs Institute for Chemical Biology, Worm Institute of Research and Medicine, Scripps Research, 10550 N Torrey Pines Road, La Jolla, CA 92037, United States. ORCID: 0000-0001-6759-4227; Email: kdjanda@ scripps.edu

\section{Authors}

Lewis D. Turner - Department of Chemistry, Scripps Research, 10550 N Torrey Pines Road, La Jolla, CA 92037, United States. ORCID: 0000-0003-0660-8247

Alexander L. Nielsen - Departments of Chemistry, Scripps Research, $10550 \mathrm{~N}$ Torrey Pines Road, La Jolla, CA 92037, United States. Current address: Institute of Chemical Sciences and Engineering, Ecole Polytechnique Fédérale de Lausanne, Lausanne, CH-1015, Switzerland. ORCID: $\underline{0000-0003-1195-0143}$

Lucy Lin - Department of Chemistry, Scripps Research, 10550 N Torrey Pines Road, La Jolla, CA 92037, United States. ORCID: 0000-0001-9546-6919

Antonio J. Campedelli - Department of Chemistry, Scripps Research, 10550 N Torrey Pines Road, La Jolla, CA 92037, United States. Current address: Eurofins Lancaster Laboratories, 2425 New Holland Pike, Lancaster, PA 17601, United States.

Nicholas R. Silvaggi - Department of Chemistry, Boston University, Boston, MA 02215, United States. Current address: Department of Chemistry and Biochemistry, University of Wisconsin, Milwaukee, WI 53211, United States ORCID: 0000-0003-0576-0714

Jason S. Chen - Automated Synthesis Facility, Scripps Research, 10550 N Torrey Pines Road, La Jolla, CA 92037, United States.

Amanda E. Wakefield - Department of Biomedical Engineering and Department of Chemistry, Boston University, Boston, MA 02215, United States. ORCID: 0000-0001-7962-2686

Karen N. Allen - Graduate Program in Molecular Biology, Cell Biology and Biochemistry and Department of Chemistry, Boston University, Boston, MA 02215, United States. ORCID: 00000001-7296-0551 


\section{Author Contributions}

L.D.T conceptualized the study; L.D.T, A.L.N., and A.C carried out chemical synthesis and characterization; L.L and A.C carried out biochemical assays and data analysis; N.R.S carried out crystallization studies and refinement; A.L.N and A.E.W performed computational modeling and calculations; J.S.C carried out and overlooked SFC; L.D.T wrote the original draft of the manuscript and all authors revised the final version; K.N.A and K.D.J acquired funding and supervised the study.

\section{Notes}

There are no conflicts of interest to declare.

\section{Acknowledgements}

The authors thank James Conley for his assistance regarding crystallography, Dr. Joseph Barbieri for providing truncated BoNT/A LC, Emily Sturgell and Brittany Sanchez for their contribution to SFC, and William H. Tepp for production of the BoNT/A holotoxin. This work was supported by the National Institutes of Health grants R01 AI153298; the Fulbright Scholar Program (A.L.N.); the Natural Sciences and Engineering Research Council of Canada PGSD3-502274 (L.L.) and the Skaggs Institute for Chemical Biology (L.L. and K.D.J.). This is Scripps Research manuscript \#XXXXX

\footnotetext{
Abbreviations

BoNT/A, botulinum neurotoxin A; CDC, Center for Disease Control and Prevention; DCHA, 2,4dichlorocinnamic hydroxamic acid; DIPEA, diisopropylethylamine; DMF, dimethylformamide; EDC, 1-ethyl-3-(3-dimethylaminopropyl)carbodiimide; FDA, Food and Drug Administration; FRET, fluorescence resonance energy transfer; HATU, 1-[bis(dimethlyamino)methylene]-1H-1,2,3triazolo[4,5-b]pyridinium 3-oxide hexafluorophosphate; HC, heavy chain; LC, light chain; MTS, methanethiosulfonate; NSF, N-ethylmaleimide sensitive factor; PDB, protein data bank; PPTS, pyridinium $p$-toluenesulfonate; SARs, structure-activity relationships; SBDD, structure-based drug design; SFC, supercritical fluid chromatography; SI, supplementary information; SNARE, NSF attachment protein factor; SNAP-25, synaptosomal-associated protein-25; TFA, trifluoroacetic acid; THP, tetrahydropyran.
} 


\section{References}

1. Collins, M. D.; East, A. K., Phylogeny and taxonomy of the food-borne pathogen Clostridium botulinum and its neurotoxins. J. Appl. Microbiol. 1998, 84, 5-17.

2. Jackson, K. A.; Mahon, B. E.; Copeland, J.; Fagan, R. P., Botulism mortality in the USA, 1975-2009. Botulinum J 2015, 3, 6-17.

3. Arnon, S. S.; Schechter, R.; Inglesby, T. V.; Henderson, D. A.; Bartlett, J. G.; Ascher, M. S.; Eitzen, E.; Fine, A. D.; Hauer, J.; Layton, M.; Lillibridge, S.; Osterholm, M. T.; O'Toole, T.; Parker, G.; Perl, T. M.; Russell, P. K.; Swerdlow, D. L.; Tonat, K. \& the Working Group on Civilian Biodefense, Botulinum toxin as a biological weaponMedical and public health management. JAMA 2001, 285, 1059-1070.

4. $\quad$ Foran, P. G.; Mohammed, N.; Lisk, G. O.; Nagwaney, S.; Lawrence, G. W.; Johnson, E.; Smith, L.; Aoki, K. R.; Dolly, J. O., Evaluation of the therapeutic usefulness of botulinum neurotoxin $\mathrm{B}, \mathrm{C} 1, \mathrm{E}$, and $\mathrm{F}$ compared with the long lasting type A - Basis for distinct durations of inhibition of exocytosis in central neurons. J. Biol. Chem. 2003, 278, 1363-1371.

5. Oliveira, M.; Mason-Buck, G.; Ballard, D.; Branicki, W.; Amorim, A., Biowarfare, bioterrorism and biocrime: A historical overview on microbial harmful applications. Forensic Sci. Int. 2020, 314, 110366.

6. Sharma, S. K.; Whiting, R. C., Methods for detection of Clostridium botulinum toxin in foods. J. Food Prot. 2005, 68, 1256-1263.

7. CDC. Home canning and botulism. https://www.cdc.gov/foodsafety/communication/homecanning-and-botulism.html (accessed 03/18/2021).

8. Samizadeh, S.; De Boulle, K., Botulinum neurotoxin formulations: overcoming the confusion. Clin. Cosmet. Investig. Dermatol. 2018, 11, 273-287.

9. $\quad$ Farag, S. M.; Mohammed, M. O.; El-Sobky, T. A.; ElKadery, N. A.; ElZohiery, A. K., Botulinum Toxin A Injection in Treatment of Upper Limb Spasticity in Children with Cerebral Palsy: A Systematic Review of Randomized Controlled Trials. JBJS Reviews 2020, 8, e0119.

10. Lipton, R. B.; Rosen, N. L.; Ailani, J.; DeGryse, R. E.; Gillard, P. J.; Varon, S. F., OnabotulinumtoxinA improves quality of life and reduces impact of chronic migraine over one year of treatment: Pooled results from the PREEMPT randomized clinical trial program. Cephalalgia 2016, 36, 899-908.

11. Oliveira, A. F. d. F.; Silva, G. A. d. M.; Almeida, D. M. X., Application of botulinum toxin to treat sialorrhea in amyotrophic lateral sclerosis patients: a literature review. Einstein (Sao Paulo) 2016, $14,431-434$.

12. Small, R., Botulinum toxin injection for facial wrinkles. Am. Fam. Physician 2014, 90, 168175.

13. Yiannakopoulou, E., Serious and long-term adverse events associated with the therapeutic and cosmetic use of botulinum toxin. Pharmacology 2015, 95, 65-69.

14. Pellizzari, R.; Rossetto, O.; Schiavo, G.; Montecucco, C., Tetanus and botulinum neurotoxins: mechanism of action and therapeutic uses. Philos. Trans. R. Soc. Lond. B Biol. Sci. 1999, 354, 259-268.

15. Rummel, A.; Karnath, T.; Henke, T.; Bigalke, H.; Binz, T., Synaptotagmins I and II act as nerve cell receptors for botulinum neurotoxin G. J. Biol. Chem. 2004, 279, 30865-30870.

16. Sun, S.; Suresh, S.; Liu, H.; Tepp, W. H.; Johnson, E. A.; Edwardson, J. M.; Chapman, E. R., Receptor binding enables botulinum neurotoxin B to sense low $\mathrm{pH}$ for translocation channel assembly. Cell Host Microbe 2011, 10, 237-247.

17. Sepulveda, J.; Mukherjee, J.; Tzipori, S.; Simpson, L. L.; Shoemaker, C. B., Efficient serum clearance of botulinum neurotoxin achieved using a pool of small antitoxin binding agents. Infect. Immun. 2010, 78, 756-763.

18. Lin, L.; Olson, M. E.; Eubanks, L. M.; Janda, K. D., Strategies to counteract botulinum neurotoxin A: Nature's deadliest biomolecule. Acc. Chem. Res. 2019, 52, 2322-2331.

19. Lin, L.; Turner, L. D.; Šilhár, P.; Pellett, S.; Johnson, E. A.; Janda, K. D., Identification of 3-hydroxy-1,2-dimethylpyridine-4(1H)-thione as a metal-binding motif for the inhibition of botulinum neurotoxin A. RSC. Med. Chem. 2021, 12, 137-143. 
20. Boldt, G. E.; Kennedy, J. P.; Janda, K. D., Identification of a Potent Botulinum Neurotoxin A Protease Inhibitor Using in Situ Lead Identification Chemistry. Org. Lett. 2006, 8 (8), 1729-1732.

21. S Šlhár, P.; Silvaggi, N. R.; Pellett, S.; Čapková, K.; Johnson, E. A.; Allen, K. N.; Janda, K. D., Evaluation of adamantane hydroxamates as botulinum neurotoxin inhibitors: synthesis, crystallography, modeling, kinetic and cellular based studies. Biorg. Med. Chem. 2013, 21, 1344-1348. 22. $\quad \mathrm{Fu}$, Z.; Chen, S.; Baldwin, M. R.; Boldt, G. E.; Crawford, A.; Janda, K. D.; Barbieri, J. T.; Kim, J.-J. P., Light chain of botulinum neurotoxin serotype A: Structural resolution of a catalytic intermediate. Biochemistry 2006, 45, 8903-8911.

23. Silvaggi, N. R.; Boldt, G. E.; Hixon, M. S.; Kennedy, J. P.; Tzipori, S.; Janda, K. D.; Allen, K. N., Structures of Clostridium botulinum Neurotoxin Serotype A Light Chain complexed with smallmolecule inhibitors highlight active-site flexibility. Chem. Biol. 2007, 14, 533-542.

24. Thompson, A. A.; Jiao, G. S.; Kim, S.; Thai, A.; Cregar-Hernandez, L.; Margosiak, S. A.; Johnson, A. T.; Han, G. W.; O'Malley, S.; Stevens, R. C., Structural characterization of three novel hydroxamate-based zinc chelating inhibitors of the Clostridium botulinum serotype A neurotoxin light chain metalloprotease reveals a compact binding site resulting from 60/70 loop flexibility. Biochemistry 2011, 50, 4019-4028.

25. Stowe, G. N.; Šilhár, P.; Hixon, M. S.; Silvaggi, N. R.; Allen, K. N.; Moe, S. T.; Jacobson, A. R.; Barbieri, J. T.; Janda, K. D., Chirality holds the key for potent inhibition of the botulinum neurotoxin serotype A protease. Org. Lett. 2010, 12, 756-759.

26. Lin, L.; Olson, M. E.; Sugane, T.; Turner, L. D.; Tararina, M. A.; Nielsen, A. L.; Kurbanov, E. K.; Pellett, S.; Johnson, E. A.; Cohen, S. M.; Allen, K. N.; Janda, K. D., Catch and Anchor Approach To Combat Both Toxicity and Longevity of Botulinum Toxin A. J. Med. Chem. 2020, 63, $11100-11120$.

27. Turner, L. D.; Nielsen, A. L.; Lin, L.; Pellett, S.; Sugane, T.; Olson, M. E.; Johnson, E. A.; Janda, K. D., Irreversible inhibition of BoNT/A protease: proximity-driven reactivity contingent upon a bifunctional approach. RSC Med. Chem. 2021. https://doi.org/10.1039/D1MD00089F

28. Le Guilloux, V.; Schmidtke, P.; Tuffery, P., Fpocket: An open source platform for ligand pocket detection. BMC Bioinformatics 2009, 10, 168.

29. Schmidtke, P.; Le Guilloux, V.; Maupetit, J.; Tufféry, P., fpocket: online tools for protein ensemble pocket detection and tracking. Nucleic Acids Res. 2010, 38, W582-589.

30. Baldwin, M. R.; Bradshaw, M.; Johnson, E. A.; Barbieri, J. T., The C-terminus of botulinum neurotoxin type A light chain contributes to solubility, catalysis, and stability. Protein Expr. Purif. 2004, 37, 187-195.

31. Shine, N. S., K., New FRET substrate for botulinum neurotoxin type A. In 7th International Conference on Basic and Therapeutic Aspects of Botulinum and Tetanus Toxins, Santa Fe, NM, USA, 2011. 


\section{For Table of Contents only}

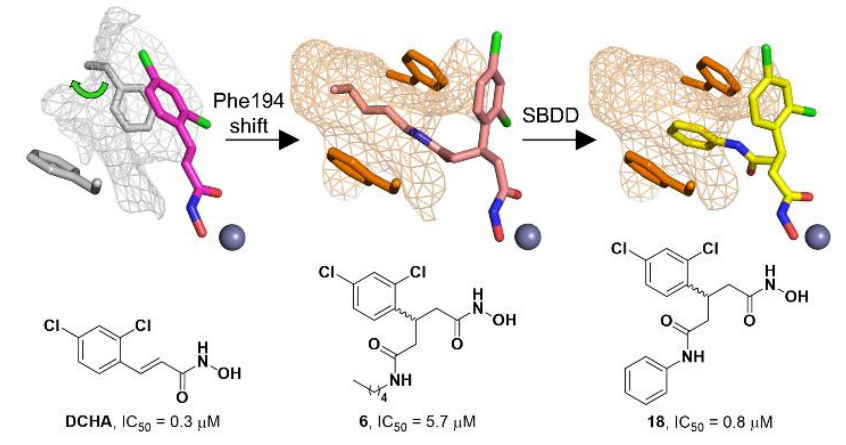

As one door closes, another one opens - The structure-guided design of inhibitors of botulinum neurotoxin protease using a new, cryptic, hydrophobic sub-pocket. 